\title{
Novel Influenza A (H1N1)-Associated Acute Necrotizing Encephalopathy: A Case Report
}

\author{
Ki Jung Kim, $\mathrm{MD}^{1}$, Eun Sook Park, $\mathrm{MD}^{1}$, Hyun Jung Chang, $\mathrm{MD}^{2}$, Miri Suh, $\mathrm{MD}^{1}$, Dong-Wook Rha, $\mathrm{MD}^{1}$ \\ ${ }^{1}$ Department and Research Institute of Rehabilitation Medicine, Yonsei University College of Medicine, Seoul; \\ ${ }^{2}$ Department of Physical and Rehabilitation Medicine, Samsung Changwon Hospital, \\ Sungkyunkwan University School of Medicine, Changwon, Korea
}

Several cases of acute necrotizing encephalopathy (ANE) with influenza A (H1N1) have been reported to date. The prognosis of ANE associated with H1N1 is variable; some cases resulted in severe neurologic complication, whereas other cases were fatal. Reports mostly focused on the diagnosis of ANE with H1N1 infection, rather than functional recovery. We report a case of ANE with H1N1 infection in a 4-year-old Korean girl who rapidly developed fever, seizure, and altered mentality, as well as had neurologic sequelae of ataxia, intentional tremor, strabismus, and dysarthria. Brain magnetic resonance imaging showed lesions in the bilateral thalami, pons, and left basal ganglia. To our knowledge, this is the first report of ANE caused by H1N1 infection and its long-term functional recovery in Korea.

Keywords H1Nl subtype influenza A virus, Viral encephalitis, Humans influenza

\section{INTRODUCTION}

Since the World Health Organization declared a global pandemic of novel influenza A (H1N1) in June 2009, the number of cases of this strain of influenza has steadily been rising. Although most cases are mild with complete and uneventful recovery, multiple cases of severe infection with complications including death have been reported. Unfavorable outcome was mostly related to

Received March 30, 2012; Accepted June 25, 2012

Corresponding author: Dong-Wook Rha

Department and Research Institute of Rehabilitation Medicine, Yonsei University College of Medicine, 50 Yonsei-ro, Seodaemun-gu, Seoul 120749 , Korea

Tel: +82-2-2228-3717, Fax: +82-2-363-2795, E-mail: medicus@yuhs.ac

(c) This is an open-access article distributed under the terms of the Creative Commons Attribution Non-Commercial License (http://creativecommons. org/licenses/by-nc/3.0) which permits unrestricted noncommercial use, distribution, and reproduction in any medium, provided the original work is properly cited.

Copyright $\odot 2013$ by Korean Academy of Rehabilitation Medicine pulmonary complications. However, a few cases with severe neurologic complications have been reported in the medical literature [1-3]. Recently, several cases of neurologic complication of H1N1 have been reported in Korea $[4,5]$. However, these cases had no neurologic sequelae on discharge and did not correspond to acute necrotizing encephalopathy (ANE).

Here, we report a 4-year-old Korean girl infected with H1N1 whose clinical course was complicated by rapid, progressive neurologic deterioration and striking magnetic resonance imaging (MRI) findings consistent with ANE.

\section{CASE REPORT}

A 4-year-old girl with normal neurodevelopmental aspects was diagnosed with $\mathrm{H} 1 \mathrm{~N} 1$ infection by real-time polymerase chain reaction test from nasopharyngeal swabs and given Tamiflu. On the following day, she was 
presented with generalized tonic-clonic type seizure and neurologic deterioration, requiring intensive care. Cerebrospinal fluid (CSF) analysis revealed an increase in protein level $(88 \mathrm{mg} / \mathrm{dL})$ and a normal glucose level (46 mg/dL), but negative results in bacterial antigen test with no pleocytosis. Gram staining and cultures of CSF and blood were negative. The C-reactive protein value was normal $(<1 \mathrm{mg} / \mathrm{L})$ and the blood leukocyte count was elevated $\left(15.69 \times 10^{3} / \mu \mathrm{L}\right)$. Serum aminotransferases was elevated (83 IU/L), but blood ammonia $(41 \mu \mathrm{g} /$
$\mathrm{dL}$ ) and renal function tests were normal. There was no definite abnormality in electroencephalogram. Initial magnetic resonance image (MRI) demonstrated enhancing lesions in the bilateral thalami, pons, and left basal ganglia that suggested sequelae of previous viral infection, which were findings compatible with ANE (Fig. 1A). Based on the CSF study, viral and bacterial encephalitis were excluded. Hypoxia, intoxication, hemolytic-uremic syndrome, metabolic disease, Leigh syndrome and other encephalopathies with thalamic involvement, such as
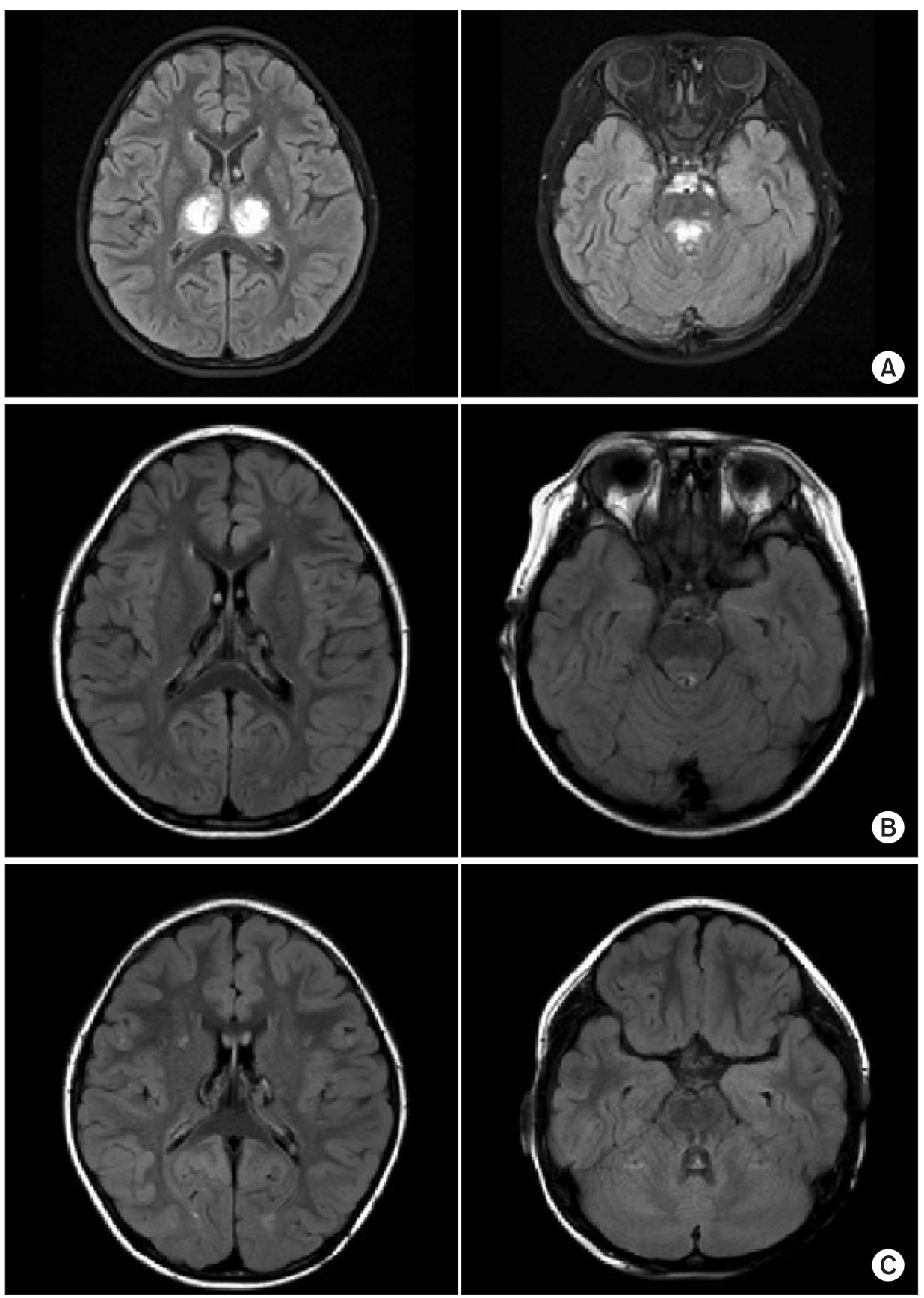

Fig. 1. (A) Brain magnetic resonance images (MRIs) taken on November 11, 2009 showing enhancing lesions in bilateral thalami, pons, and left basal ganglia, suggesting sequelae of previous viral infection in T2-weighted images. (B) Brain MRIs taken on January 8,2010 showing a decrease in the extent of lesions involving bilateral thalami and brainstem with remaining hemorrhagic residues. (C) Brain MRIs taken on April 29, 2010 showing no interval change of hemorrhagic residues in dorsal brain stem and bilateral thalami. 
Wernicke encephalopathy, thalamic infarction, and deep cerebral vein thrombosis were excluded by laboratory data, clinical course, and MRI findings. As a result, ANE caused by H1N1 infection was diagnosed according to the previously suggested diagnostic criteria $[6,7]$. One month after disease onset, the patient was transferred to the department of rehabilitation medicine. Although the precise muscle strength could not be measured due to altered mental status and young age, antigravity muscle activities were observed in all four extremities. Neurologic signs implicating upper motor neuron damages, such as Hoffmann's sign, ankle clonus, Babinski's sign or hyperactive deep tendon reflexes were not observed. She could not sit, stand, walk, or grasp an object. She could make sounds, but could not speak words. Strabismus due to the left abducens nerve palsy was observed (Fig. 2A). The Gross Motor Function Measure (GMFM)-88 was 15.16\%, and the Functional Independence Measure for children (WeeFIM) score was 18 of 126 . Her social age (SA) was 1.08 years and social quotient (SQ) was 22.73. On the 18Ffluorodeoxyglucose positron emission tomography brain scan, glucose uptake increased diffusely in the bilateral basal ganglia and mildly in the bilateral thalami, but decreased diffusely in the bilateral cerebral hemispheres (Fig. 3). Physical, occupational, and speech therapy ensued. On discharge, 77 days after onset, she was able to walk on an even surface under supervision and her GMFM-88 was $78.81 \%$. The SA was 3.0 years and SQ was 62.11. Follow-up MRI showed a decrease in the extent of the lesions involving the bilateral thalami and brainstem, with remaining hemorrhagic residue (Fig. 1B).

The patient continued rehabilitative management via the outpatient clinic. Four months after initial evaluation, she could walk independently and ascend and descend stairs, but showed ataxia. She mainly used her right hand and suffered from intention tremor in the left hand. Although the degree of left abducens nerve palsy was improved, it is still present (Fig. 2B). The International Cooperative Ataxia Rating Scale [8] was 50 and Scale for the Assessment and Rating of Ataxia [9] was 14, which corresponds to moderate ataxia. Her GMFM-88 had improved to $92.59 \%$ and the WeeFIM score was 70 ; SA was 4.07 years and SQ was 91.08. A follow-up MRI taken on April 29, 2010 showed no interval change of the hemorrhagic
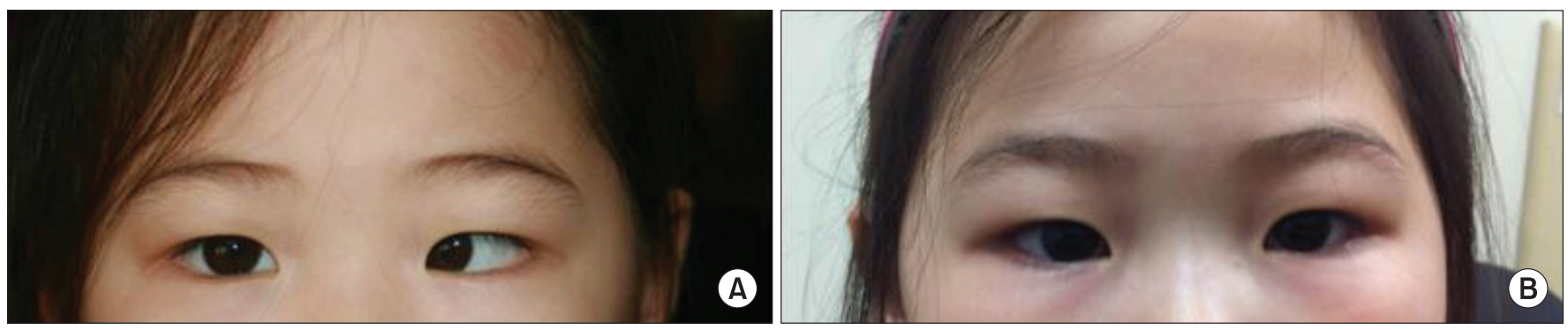

Fig. 2. Left abducens nerve palsy was observed on January 11, 2010 (A) and was improved on April 15, 2010 (B).
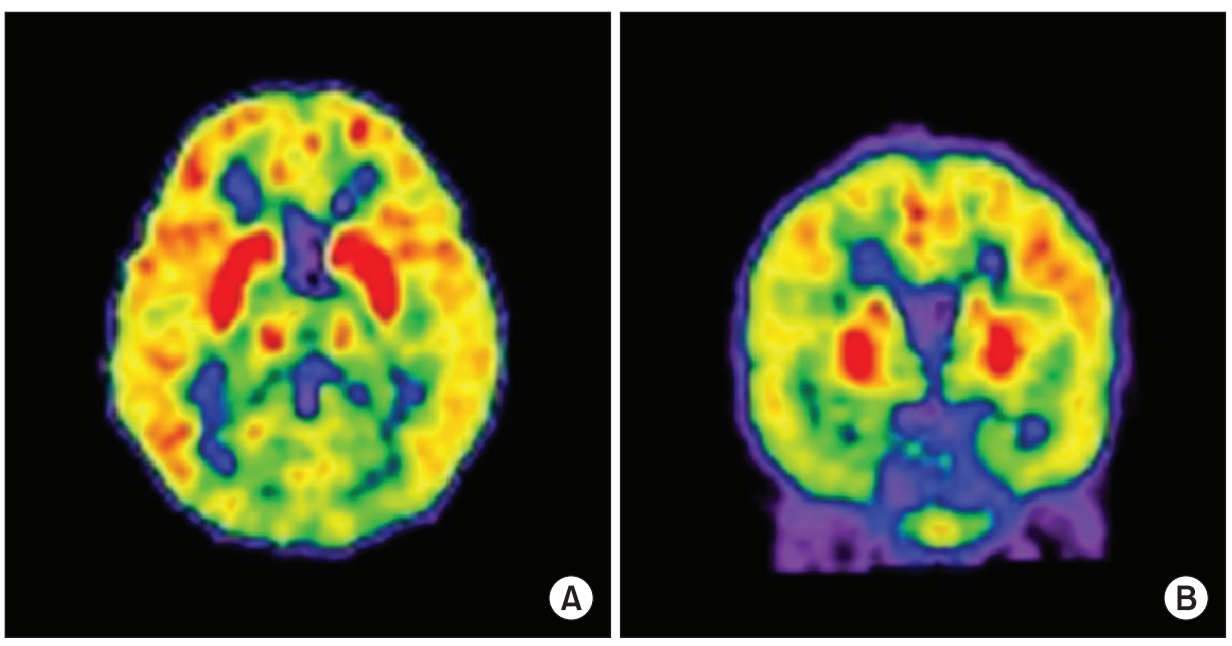

Fig. 3. Fluorodeoxyglucose-positron emission tomography brain scan taken on December 28, 2009. Glucose uptake increased diffusely in bilateral basal ganglia and mildly in bilateral thalami, but decreased diffusely in bilateral cerebral hemispheres in both transverse (A) and coronal (B) sections. 
residue in the dorsal brain stem and bilateral thalami (Fig. 1C). Intentional tremor of the left hand, ataxic walking pattern, and left abducens nerve palsy were still observed 2 years after onset.

\section{DISCUSSION}

Influenza A viruses represent a continuous pandemic threat. In April 2009, a novel influenza A virus, the socalled swine-origin influenza A (H1N1) virus, was isolated in Mexico, and several cases of ANE caused by H1N1 infection were recently reported [1-3].

ANE is a clinical, pathologic entity, which was first described by Mizuguchi et al. [6] in 1995. The proposed diagnostic criteria for ANE include acute onset encephalopathy with rapid, progressive neurologic deterioration, seizures and symmetric multifocal brain lesions. Protein is commonly increased without pleocytosis in CSF and serum aminotransferase is elevated without serum ammonia elevation. Similar diseases, such as hypoxia, intoxication, hemolytic-uremic syndrome, metabolic disease, and neurodegenerative disorder, should also be excluded $[6,7]$. ANE has been predominantly reported in children of East Asian descent, including Japan, Taiwan, and Korea, although sporadic cases have been documented worldwide.

The exact etiology of ANE is unknown. Influenza A virus, mycoplasma, herpes simplex virus, and human herpes virus- 6 have been reported as common causative agents, acting directly or through an immune-mediated mechanism [7]. The first symptom of ANE is brain dysfunction, such as convulsion, impairment of consciousness, and vomiting [7]. At the advanced stage of ANE, hypotension, apnea, and hypotonia are seen [7]. There is no specific therapy for ANE, and the mortality of ANE is reported as approximately $30 \%[6,7]$, but supportive care, steroid pulse therapy, and antiviral therapy have been used to treat some cases in Japan [10]. Early steroid treatment showed better outcome in children with ANE without brainstem lesions [10].

Two cases of ANE caused by H1N1 infection were reported in the United States: a 12-year-old Caucasian girl and a 7-year-old girl, who progressed to brain death $[1,2]$. In Italy, a 2-year-old girl from Eastern Europe with sequelae of a divergent strabismus of the left eye and gait disturbance was reported [3].
In the present case, the symmetric brain lesions involving the thalami, basal ganglia, and pons, combined with fever, seizure, intentional tremor, and ataxia were consistent with ANE, as previously reported by Mizuguchi et al. $[6,7]$, and serial MRI revealed gradual resolution of edema and cavitations of the thalami and brain stem, as typically described in patients with ANE. Viral encephalitis could be excluded because the protein level was elevated without pleocytosis in CSF analysis.

This patient showed motor weakness, ataxia, intention tremor in the left hand, and strabismus caused by abducens nerve palsy. In pontine lesions, ataxia is accompanied by weakness and pyramidal tract signs as part of an ataxic hemiparesis syndrome. Strabismus caused by abducens nerve palsy can be observed in pontine lesions because the abducens nerve originates from the pons in the floor of the fourth ventricle. This nerve descends through the pontine tegmentum and emerges at the caudal pons. Pontine hemorrhagic lesions were thought to result in ataxia and strabismus in this patient.

On the first evaluation, 1 month after onset, the patient could not sit, grasp an object, or speak words, but showed rapid functional recovery. Forty days later, she was able to walk on an even surface under supervision. However, cerebellar symptoms, including ataxic dysarthria and left abducens nerve palsy were still observed four months later. To our knowledge, this is the first case of ANE caused by H1N1 infection and its long-term functional recovery reported in Korea.

\section{CONFLICT OF INTEREST}

No potential conflict of interest relevant to this article was reported.

\section{REFERENCES}

1. Lyon JB, Remigio C, Milligan T, Deline C. Acute necrotizing encephalopathy in a child with H1N1 influenza infection. Pediatr Radiol 2010;40:200-5.

2. Martin A, Reade EP. Acute necrotizing encephalopathy progressing to brain death in a pediatric patient with novel influenza A (H1N1) infection. Clin Infect Dis 2010;50:e50-2.

3. Mariotti P, Iorio R, Frisullo G, Plantone D, Colantonio $\mathrm{R}$, Tartaglione T, et al. Acute necrotizing encephalopa- 
thy during novel influenza A (H1N1) virus infection. Ann Neurol 2010;68:111-4.

4. Kim RH, Kim YM, Park SE, Kim HY, Lee YJ, Kim TH, et al. Neurologic complication of novel influenza A (H1N1) virus infection from 2009-2011. J Korean Child Neurol Soc 2011;19:54-60.

5. Choi SY, Jang SH, Kim JO, Ihm CH, Lee MS, Yoon SJ. Novel swine-origin influenza A (H1N1) viral encephalitis. Yonsei Med J 2010;51:291-2.

6. Mizuguchi M, Abe J, Mikkaichi K, Noma S, Yoshida K, Yamanaka T, et al. Acute necrotising encephalopathy of childhood: a new syndrome presenting with multifocal, symmetric brain lesions. J Neurol Neurosurg Psychiatry 1995;58:555-61.

7. Mizuguchi M. Acute necrotizing encephalopathy of childhood: a novel form of acute encephalopathy prevalent in Japan and Taiwan. Brain Dev 1997;19:81-
92.

8. Trouillas $\mathrm{P}$, Takayanagi $\mathrm{T}$, Hallett $\mathrm{M}$, Currier RD, Subramony $\mathrm{SH}$, Wessel $\mathrm{K}$, et al. International cooperative ataxia rating scale for pharmacological assessment of the cerebellar syndrome: the Ataxia Neuropharmacology Committee of the World Federation of Neurology. J Neurol Sci 1997;145:205-11.

9. Schmitz-Hubsch T, du Montcel ST, Baliko L, Berciano J, Boesch S, Depondt C, et al. Scale for the assessment and rating of ataxia: development of a new clinical scale. Neurology 2006;66:1717-20.

10. Okumura A, Mizuguchi M, Kidokoro H, Tanaka M, Abe S, Hosoya M, et al. Outcome of acute necrotizing encephalopathy in relation to treatment with corticosteroids and gammaglobulin. Brain Dev 2009;31:2217. 